\title{
La fuerza de los lazos: una exploración teórica y empirica de sus múltiples significados*
}

\author{
The strength of ties: a theoretical and empirical exploration \\ of its multiple meanings \\ Irene Cruz Gómez Y y Joan Miquel Verd \\ Universidad Autónoma de Barcelona \\ irene.cruz@uab.cat (ESPAÑA)
}

Recibido: 21.01 .2012

Aceptado: 16.05.2013

\section{RESUMEN}

La fuerza del vínculo es una de las características recurrentemente estudiadas en las redes personales. Es un dato aparentemente sencillo de generar, con una potencial capacidad predictiva sobre los intercambios dentro de la relación y de la posición del contacto dentro de la red. Sin embargo, la gran diversidad de indicadores que se emplean en la literatura para medir esta fuerza del vínculo da fe de la vaguedad en la definición del concepto y las consecuentes dificultades para operativizarlo, dando lugar a resultados ambivalentes en su observación empírica. Con este trabajo de revisión teórica, metodológica y empírica se pretende ofrecer una base sobre la cual contribuir a la delimitación del concepto. Se propone una dimensionalización teórica de la noción, distinguiendo sus aspectos expresivos, sociales y estructurales, y a continuación se compara el comportamiento de diversos indicadores de las distintas dimensiones empleando una muestra de 441 redes personales y 13.230 relaciones provenientes de una encuesta realizada en Cataluña entre 2009 y 2010.

* Este artículo es el resultado de los anàlisis desarrollados en el seno del proyecto CASREDIN (Estudio comparado de casos sobre la influencia mutua entre capital e integración sociales y la inserción, estabilidad, promoción y cualificación en el empleo), financiado por el Ministerio de Educación y Ciencia en el marco del VI Plan Nacional de Investigación Científica, Desarrollo e Innovación Tecnológica 2008-2011 (contrato número CSO2008-01470).

${ }^{1}$ Con el apoyo del Comissionat per a Universitats i Recerca del DIUE de la Generalitat de Catalunya y del Fondo Social Europeo.

EMPIRIA. Revista de Metodología de Ciencias Sociales. N. ${ }^{\circ}$ 26, julio-diciembre, 2013, pp. 149-174. ISSN: 1139-5737, DOI: 10.5944/empiria.26.7156 


\title{
PALABRAS CLAVE
}

Fuerza de los lazos débiles, puente, operativización, homofilia, red personal.

\begin{abstract}
The strength of a tie is feature commonly studied in the analysis of personal networks. It is data apparently simple to create, that offers a great predictive potential on the exchanges that take place within a relationship, and of its position within the network. However, the wide diversity of indicators that can be found in the literature show evidence of the vagueness in the definition of the concept and the subsequent difficulty to operationalize it, returning mixed results in empirical observation. With this theoretical, methodological and empirical revision, the authors aim to contribute to the definition of the concept. A theoretical dimensionalization of the concept is proposed, distinguishing the expressive, social and structural aspects of the notion. A comparison of various indicators of the different dimensions is offered, using a sample of 441 personal networks and 13,230 relationships, from a survey conducted in Catalonia between 2009 and 2010.
\end{abstract}

\section{KEYWORDS}

Strength of weak ties, bridge, operationalization, homophily, personal network

\section{INTRODUCCIÓN}

La fuerza del vínculo es una de las características recurrentemente estudiadas en las redes personales. Es una información aparentemente fácil de generar, que a su vez informa sobre una de las dimensiones más relevantes en el estudio de las relaciones interpersonales. También tiene una gran capacidad predictiva sobre los contenidos y los intercambios que potencialmente pueden darse dentro de una relación determinada (Granovetter, 1973; Wellman, 1982; Lin, Vaighn y Ensel, 1981). Tomando como referente el trabajo de Mark Granovetter (1973), la fuerza del vínculo es entendida también como un dato que informa sobre la posición estructural de los contactos, a partir de la noción de las tríadas prohibidas².

Sin embargo, pese al evidente interés que encierra esta característica de las relaciones, el concepto de "fuerza del vínculo" sigue siendo relativamente abstracto, $\mathrm{y}$ hace referencia a aspectos muy diferentes de las relaciones (como la intensidad del vínculo definido subjetivamente, el tiempo invertido en la relación o el intercambio de apoyo) que no son necesariamente equivalentes. La gran diversidad

${ }^{2}$ La noción de tríada prohibida se explica en el primer epígrafe del artículo. 
de indicadores que se emplean en la literatura para medir este concepto da fe de la vaguedad en la definición del concepto y las consecuentes dificultades para operativizarlo, dando lugar a resultados ambivalentes en su observación empírica.

En este contexto, el artículo identifica tres dimensiones diferentes sobre las que puede versar la noción de "fuerza del vínculo". A esta revisión teórico-metodológica se añade una revisión de un buen número de indicadores desarrollados en la literatura como modo de operativizar la noción, lo cual permite identificar algunas de las relaciones entre las diferentes dimensiones a la que hace referencia el concepto.

A nivel empírico, el artículo pretende comparar el comportamiento de varios indicadores asociados a la fuerza del vínculo: los clásicos definidos por Granovetter (la intensidad afectiva, el intercambio de distintas formas de apoyo, la duración de la relación), y otros empleados a menudo en la literatura (la mutiplexidad, la homofilia y la transitividad). Peter Marsden y Karen Campbell realizaron una comparación entre diversas medidas de la fuerza del vínculo en Measuring Tie Strength (1984). Sus resultados sugieren que la definición subjetiva de la intensidad de la relación es el mejor de los indicadores. Sin embargo, este ejercicio fue realizado exclusivamente con datos de vínculos fuertes (los tres mejores amigos de la persona entrevistada), por lo que su operativización de la "fuerza de los lazos" puede verse sesgada hacia los lazos fuertes.

A diferencia de los datos empleados por Marsden y Campbell (1984), en esta propuesta se trabaja con una muestra de redes personales que incluye diversos tipos de vínculos de distinta intensidad. Los datos provienen de una encuesta de redes personales aplicada a una muestra de 441 individuos, realizada entre 2009 y 2010, en tres poblaciones catalanas. Las redes se han construido siguiendo el método McCarty (2002), es decir, con un número fijo de contactos. De acuerdo con el autor, treinta es una cantidad de relaciones capaz de recoger no sólo a las personas más próximas a ego (la persona encuestada), sino también otras relaciones menos cercanas, que se entiende que son los vínculos más débiles. Al disponer de una diversidad de contactos de distintas intensidades, nuestra muestra presenta un interés especial para realizar el ejercicio propuesto. Además, la base de datos utilizada permite analizar la fortaleza de los vínculos en relación con indicadores de homofilia y multiplexidad (o polivalencia), un análisis no desarrollado por Marsden y Campbell.

Por otra parte, el análisis realizado permite también contestar a las siguientes preguntas: a) ¿Cuáles son las características de los alteri con los que se mantienen "vínculos débiles", y qué relaciones mantienen con los ego?, y b) ¿Cómo se relaciona la proximidad expresiva con los otros indicadores de la fuerza del lazo, si consideramos también los lazos débiles?

El artículo se estructura en cinco apartados principales. En primer lugar se expone brevemente la noción de fuerza del vínculo presentada por Granovetter en su artículo de 1973. A continuación, se propone una dimensionalización conceptual, en la que se ponen de manifiesto las distintas aproximaciones posibles sobre el concepto teórico de fuerza del vínculo, y se revisan las operativizaciones hechas por otros autores en sus trabajos. Seguidamente, en el tercer apartado, se expone la metodología seguida, y a continuación (cuarto apartado) se realizan 
una serie de análisis comparando indicadores distintos para cada dimensión teórica y se detallan las relaciones entre las distintas dimensiones e indicadores analizados. Finalmente se presentan las conclusiones alcanzadas.

\section{LA FUERZA DE LOS LAZOS DÉBILES: UNA NOCIÓN CONTROVERTIDA}

La fuerza de los lazos es una de las características más frecuentemente observadas en los análisis de redes, y particularmente al estudiar redes personales. Si bien su uso precede a la propuesta de Mark Granovetter sobre la fuerza de los lazos débiles (1973), el interés sobre la medida creció significativamente a partir de esta idea, y su desarrollo conceptual es deudor en gran parte de su planteamiento original. Resumidamente, la tesis sobre la fuerza de los lazos débiles sugiere que este tipo de contactos suponen una buena aproximación a los bridges o puentes en una red, ya que las personas con quienes tenemos una relación menos cercana suelen pertenecer a círculos socialmente distintos de los que frecuentamos en nuestras relaciones cotidianas, y consecuentemente, disponen de informaciones distintas y nuevas en relación con las que maneja nuestro entorno, lo que puede suponer una ventaja comparativa.

En la teoría de redes sociales, un puente (bridge) es un vínculo que une dos partes de una red que de otro modo serían componentes diferenciados. Es decir, es el único vínculo entre los dos sectores. Un puente local, estrictamente, es el camino más corto entre dos sectores de una red unidos en algún otro punto (véase la figura 1), sin ningún vecino en común entre los extremos del puente (caso de los nodos A y B en la figura 1).

Figura 1. Puentes locales

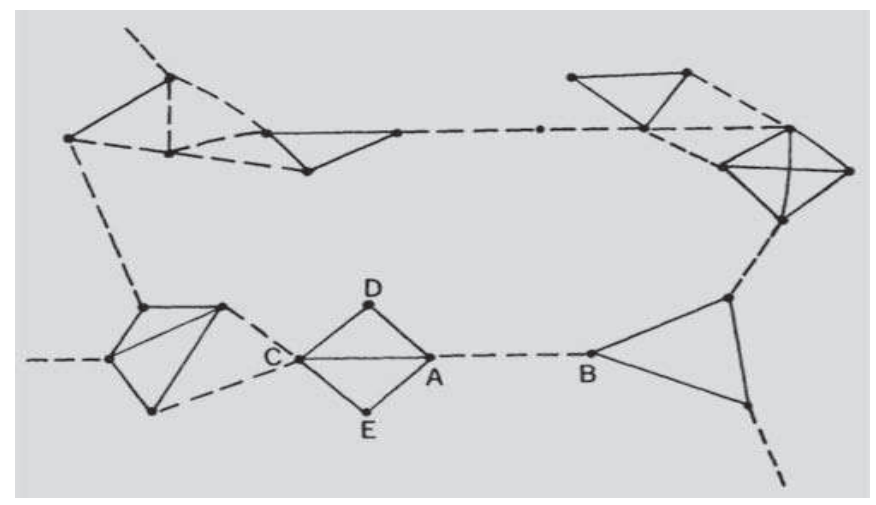

Fuente: Granovetter, 1973 (Fig. 2).

EMPIRIA. Revista de Metodología de Ciencias Sociales. N. ${ }^{\circ}$ 26, julio-diciembre, 2013, pp. 149-174. ISSN: 1139-5737, DOI: 10.5944/empiria.26.7156 
El cálculo de los puentes en una red es una información mucho más costosa de observar y generar que la fuerza del vínculo. Por ello, la propuesta de Granovetter suscitó rápidamente mucho interés. Su teoría se basa en la noción de las tríadas prohibidas (Granovetter, 1973). La idea de tríada prohibida sintetiza la dificultad para un agente de mantener dos relaciones fuertes como componentes aislados. Por ejemplo, como se observa en la figura 2, si A mantiene un vínculo fuerte con B y con C, hay más probabilidades de las esperadas en condiciones de aleatoriedad de que exista por lo menos un vínculo débil entre B y C (Rapoport, 1957). Consecuentemente, los lazos fuertes nos unen redundantemente a personas que tienen relación con nuestro entorno y, en cambio, los lazos débiles tienen mayores probabilidades de ser puentes (bridges o local bridges) hacia otros entornos sociales donde se mueven otros conjuntos de informaciones. Tanto el concepto de "tríadas prohibidas" como el de "puente" se apoyan sobre la noción de transitividad, que hace referencia a la reciprocidad en las relaciones dentro de una tríada (Hanneman y Riddle, 2005).

\section{Figura 2. Tríada prohibida}

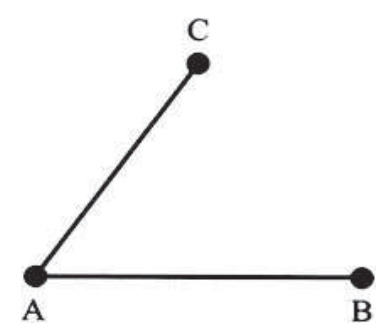

Fuente: Granovetter, 1973 (Fig. 1)

El vínculo que establece Granovetter entre la existencia de puentes en una red social y la ventaja que supone el uso instrumental de la información que por ellos circula ha sido cuestionado por diferentes autores. Son tres las principales críticas que se han formulado. En primer lugar se ha planteado que no es tan relevante la fuerza de la relación ni su posición en el conjunto de relaciones, como el estatus del contacto, puesto que las personas en posiciones jerárquicamente superiores dentro de la estructura social, tienen acceso a mayores recursos (Lin, 1999a, 2000, 2001). En segundo lugar, se ha planteado que los contactos del mundo laboral, que son los que pueden tener informaciones más relevantes para el acceso al empleo, tienden a ser relaciones más débiles que las familiares y las de amistad, y que por lo tanto, habría una relación espuria entre la fuerza del vínculo y el acceso al empleo (Forsé, 1997). Por último, se ha planteado que el contacto con vínculos débiles depende en gran medida del contexto sociopolítico, y por lo tanto sus conclusiones son difícilmente generalizables a cualquier entorno. Por ejemplo, en Alemania del Este, después de la caída del muro, las relaciones con contactos débiles son vistas con desconfianza, como riesgos 
potenciales, más que como fuentes de recursos (Völker y Flapp, 1995; citados por de Federico, 2011).

La discusión teórica en torno a las ventajas que puede suponer para un individuo contar en su red de contactos con lazos débiles ha ido acompañada por la disparidad en los planteamientos que han pretendido contrastar empíricamente esta tesis. Esta diversidad de aproximaciones empíricas obedece, en buena medida, a la multidimensionalidad de la definición ofrecida por el propio Granovetter y a las dificultades que supone operativizar las nociones de "lazo fuerte" y "lazo débil". Sin duda, estos diferentes modos de medir la "fuerza de los lazos" han complicado aún más el debate en torno a la fuerza de los lazos débiles. Es en este debate metodológico en el que quiere centrarse el artículo. Para ello, en el siguiente apartado se abordan, primero, las múltiples dimensiones que alberga la noción de "fuerza de los lazos" y, después, los diferentes indicadores que se han utilizado en la investigación empírica.

\section{LAS MÚLTIPLES CARAS DE "LA FUERZA DE LOS LAZOS" UNA NOCIÓN EMPÍRICAMENTE MULTIDIMENSIONAL Y DIFÍCIL DE OPERATIVIZAR}

A pesar del aparente avance que supuso la propuesta de Granovetter respecto al cómputo más complejo de los puentes, la operativización de la noción de fortaleza de un lazo no está exenta de problemas. En el artículo de Granovetter de 1973 se definía la fuerza del vínculo como "una combinación (probablemente lineal) de la cantidad de tiempo, de la intensidad emocional, de la intimidad (confianza mutua) y de los servicios recíprocos que caracterizan al lazo" (1973: 1361, traducción propia del original en inglés). Y sin embargo, el indicador que él empleó en su análisis de las promociones laborales de directivos estadounidenses (Granovetter, 1974) fue uno mucho más sencillo: la frecuencia de contacto. Ello muestra que tanto la noción directamente vinculada a los puentes (a través de las tríadas prohibidas), como la que entiende un lazo débil como un constructo vinculado a la intensidad emocional, la intimidad, la duración de la relación y los servicios recíprocos, presentan dificultades en su medición empírica.

Peter Marsden y Karen Campbell, en el artículo Measuring Tie Strength (1984), son los autores que con mayor detalle han analizado las dificultades que supone la medición de la fuerza de los vínculos, fruto tanto del carácter multidimensional de la definición que ofrece Granovetter como de sus dificultades de operativización. Estas dificultades han dado pie al uso de una gran diversidad de indicadores en la literatura, relativos tanto a características del ego, a la red social o a la relación entre los nodos.

Marsden y Campbell (1984) se formulaban la pregunta sobre cuál es la mejor medida de la fuerza de los lazos. Para contestarla, se plantearon el ejercicio de "construir y validar" distintas medidas de la fuerza del vínculo, empleando fundamentalmente los indicadores originalmente sugeridos por Granovetter (la cantidad de tiempo, la intensidad emocional, la confianza mutua y los servicios recíprocos). También se valieron de otras medidas habitualmente empleadas hasta ese momento 
en la literatura especializada, que en el fondo son pequeñas adaptaciones de los indicadores originales: amplitud de las temáticas discutidas, homofilia/distancia social, tiempo de conocimiento y frecuencia de contacto. Sin embargo, hay que tener en cuenta que Marsden y Campbell emplearon datos sobre los tres mejores amigos de las personas encuestadas; es decir, sólo trabajaron con información de lazos fuertes. Entre sus conclusiones destacan: a) la distinción entre lo que son propiamente indicadores de la medida (parte constitutiva de la fuerza del vínculo, por ejemplo la intensidad emocional ) y lo que denominan "predictores" (aspectos relacionados pero no constitutivos de la fuerza del lazo, por ejemplo, si la relación que une a dos nodos es de parentesco, amistad, conocimiento, etcétera); b) la afirmación de que el indicador sobre la cercanía afectiva o la intensidad emocional es la mejor medida entre todas las contrastadas; y finalmente c) que los predictores, como por ejemplo el tipo de relación entre los nodos, no son buenas medidas del fenómeno.

Por otro lado, Marsden y Campbell proponen diferenciar (1984: 482) "dos aspectos" distintos en las características de la definición ofrecida por Granovetter: un aspecto relacionado con el tiempo invertido en la relación y otro aspecto que tendría que ver con la profundidad de la relación. Es decir, se podría considerar como próxima tanto la relación con una persona con la que se pasa mucho tiempo en común como con la que se comparte mucha confianza y/o intensidad afectiva. De modo inverso, se podría considerar como un lazo débil tanto a una persona con la que tenemos un contacto muy esporádico como una con la que mantenemos una relación muy superficial.

Partiendo de estas ideas iniciales de Marsden y Campbell (1984), junto con las reflexiones provenientes de otros autores, presentamos a continuación una clasificación teórica de las posibles dimensiones a las que hace referencia la noción de "fuerza de un lazo". La clasificación que presentamos distingue tres grandes dimensiones en el concepto, a las que hemos llamado dimensión expresiva, dimensión estructural, y dimensión social. Además, se proponen tres sub-dimensiones de la dimensión expresiva.

A pesar del gran número de referencias en la literatura a la fuerza de los vínculos, son escasos los trabajos que han intentado identificar las distintas dimensiones del concepto. En nuestro caso, la clasificación que proponemos es el resultado de la revisión de numerosos artículos que han trabajado teórica y/o empíricamente la noción de "fortaleza de los lazos", entre los cuales destacan las obras clásicas de Granovetter (1973; 1974; 1983), Friedkin (1980), Lin, Vaighn y Ensel (1981), Burt (1982), Wellman (1982), Marsden y Campbell (1984) y McPherson, Popielarz y Drobnic (1992). Esta clasificación se empleará al final de este apartado 2 para revisar la gran diversidad de indicadores utilizados en la literatura, y más adelante, en la sección destinada al análisis empírico, para estructurar la comparación de los resultados obtenidos con los distintos indicadores aplicados a nuestra base de datos.

\section{Dimensión Expresiva}

Esta sería la dimensión de la fuerza del vínculo relacionada con su carga emotiva, es decir, con la valoración que el entrevistado hace de la relación en términos emocionales. Tiene abundantes indicadores para medir los lazos fuertes, las relaciones cerca- 
nas y de confianza. Sin embargo, las relaciones débiles tienden a definirse simplemente por la ausencia de todo lo anterior, su caracterización es mucho menos refinada.

Los indicadores que se emplean en relación a esta dimensión expresiva de la fuerza del vínculo acostumbran a hacer referencia a nociones de a) la intensidad de la relación a nivel afectivo, b) el tipo de apoyos que se intercambian, o c) el tiempo invertido. Estas dos últimas nociones (los apoyos intercambiados y el tiempo invertido) podrían considerarse como dimensiones independientes de la expresividad. Sin embargo, el uso que se da a estos indicadores en la literatura a partir de la propia definición de Granovetter (1973) suele ir ligado a la expresividad de las relaciones, por ejemplo, entendiendo que las relaciones en las que se intercambia apoyo emocional tienen un componente afectivo más fuerte, o que esta intensidad disminuye en las relaciones con las que se pasa menos tiempo (véanse las aportaciones a este respecto de Wellman, 1990). Por esta razón en la tabla 1 se han considerado como sub-dimensiones de la dimensión expresiva, junto con la vinculada a la intensidad de la relación.

Por otro lado, podemos distinguir los indicadores en función de si la valoración de la fuerza del vínculo parte de la definición de la persona entrevistada (subjetivos), o si es el investigador quien establece la diferenciación a partir de un hecho más o menos objetivable u observable (objetivos). Esta distinción también se recoge más adelante en la tabla 2.

Tabla 1. Indicadores de la fuerza del vínculo en la dimensión expresiva

\begin{tabular}{ccc}
\hline Sub-dimensión & Indicador Subjetivo & Indicador Objetivo \\
\hline Intensidad de la relación & $\begin{array}{c}\text { Valoración de la intensidad } \\
\text { (escala) }\end{array}$ & Diversidad de temas discutidos \\
& Nivel de confianza (escala) & \\
$\begin{array}{c}\text { Intercambio de apoyo en } \\
\text { la relación }\end{array}$ & $\begin{array}{c}\text { Intercambio de apoyo emocional } \\
\text { Tiempo invertido en la } \\
\text { relación }\end{array}$ & Extensión de la polivalencia \\
& & Duración de la relación \\
& & Frecuencia de contacto \\
\hline
\end{tabular}

Fuente: Elaboración propia.

\section{Dimensión Estructural}

En esta dimensión de la fuerza de los lazos, las relaciones se definen por el lugar que ocupan dentro de la red más amplia de contactos, prescindiendo de las valoraciones que los agentes hacen sobre las mismas. Es el sentido más literal de la idea de puente (bridge), aunque también se emplea para valorar la fuerza del vínculo. En cierto modo, la línea de investigación de Ronald Burt sobre los agujeros estructurales $(1982,1992)$ seguiría este planteamiento.

En esta dimensión, un lazo fuerte es aquel densamente conectado con el resto de contactos de ego, formando una red "close-knit" o "tupida" (Bott, 1957). 
En otros términos, un lazo fuerte es el que forma parte de una o varias tríadas transitivas, es decir, de subgrupos en los que sus relaciones están en contacto. En la medida en que los círculos sociales de dos personas se superponen, su relación será fuerte porque está encastrada (embedded) (Granovetter, 1992), independientemente de su confianza, apoyo o intensidad. También podremos considerar que ambos tendrían acceso a circuitos de información redundante. Por otro lado, los lazos débiles serían aquellos con quienes se comparten los mínimos contactos, que hacen de puente entre el círculo inmediato de ego y otras esferas sociales.

\section{Dimensión Social}

En esta dimensión se toma la distancia social como medida de la fuerza de los lazos. Su uso se fundamenta en el supuesto (véase Lin, 2001) de que los lazos fuertes conectan a gente socialmente homogénea, y los débiles a gente socialmente heterogénea. Es la misma idea que se halla detrás de los conceptos de capital social bonding y bridging tal y como se emplean en Atria (2003), Woolcock y Narayan (2000) o Lozares et al. (2011). En Marsden y Campbell (1984) los indicadores de homofilia/distancia social son tratados como predictores y no como indicadores de la fuerza del vínculo. Por lo tanto, bajo la perspectiva de Marsden y Campbell, la homo/heterogeneidad social sería una aproximación y no un indicador en sí mismo de la fuerza del vínculo (el cual, a su vez, se considera un predictor de la noción de puente). Sin embargo, cabe pensar que la idea de la distancia social tiene cierta entidad propia para definir la fuerza del vínculo y para identificar la existencia de puentes, si tomamos en consideración las revisiones y aportaciones de Lin, Ensel y Vaughn (1981) a este campo. Como se ha señalado anteriormente, estos autores plantean que no se trata sólo de a quién conoces, sino también, o sobre todo, de dónde están socialmente situadas esas personas. Entonces, en la dimensión social de la fuerza de los lazos, un lazo fuerte sería una persona atributivamente similar, que debido a sus características socio-demográficas, ocupa un lugar parecido dentro de la estructura social, y por lo tanto, tiene acceso a focos de información relativamente equivalentes. Por otra parte, un lazo débil sería aquel que, por sus características socio-demográficas, ocupa una posición jerárquicamente diferente dentro de la estructura social, teniendo acceso a recursos substancialmente distintos.

Estrictamente, en la formulación de Lin (1999a, 2001), los lazos homófilos son lazos fuertes porque el autor asimila "acción expresiva" e interacción entre individuos con recursos (personales y sociales) similares. A esta idea opone la interacción entre personas de diferente estatus y recursos, es decir, la interacción heterófila, la cual asimila a la "acción instrumental", basada en la obtención de recursos por medio del capital social. Es decir, el autor equipara los lazos homófilos a los lazos fuertes por medio de acotar la interacción entre similares a la acción expresiva, en una comprensión muy laxa del término "acción expresiva". El autor plantea este modelo dentro de su teoría del capital social, que en su formulación consta de 4 elementos que explican el efecto positivo de los recursos en las redes sobre las acciones de los individuos: la circulación de la información, la influencia sobre los agentes, las credenciales sociales y el refuerzo sobre la identidad y sobre el reconocimiento social (Lin, 1999b). De este planteamiento puede desprenderse que, si bien los tres 
últimos elementos (influencia, credenciales y reconocimiento) son recursos que circulan fundamentalmente entre los vínculos fuertes, la fuerza de los lazos débiles recae principalmente en la capacidad de controlar la circulación de la información en condiciones de mercado imperfecto (o circulación imperfecta de la información).

La homofilia con la que Lin trabaja es la de estatus social, concretamente con la categoría profesional de los entrevistados. En las páginas siguientes trabajaremos con el mismo indicador de homofilia. Sin embargo, muchas otras formas de homofilia serían concebibles, de hecho, tantas como características fueran atribuibles a una persona. Y si bien es razonable pensar que el hecho de compartir gustos, creencias o rasgos sociales con una persona aumenta las probabilidades de que la relación sea más fuerte y estable (Cohen, 1977; Kandel, 1978; Mc Pherson, SmithLovin y Cook, 2001), queda por revisar la hipótesis específica sobre el efecto de la homofilia de estatus sobre la fuerza del vínculo.

\section{APROXIMACIONES EMPÍRICAS A "LA FUERZA DE LOS LAZOS" EN LA LITERATURA}

A pesar de las recomendaciones que pueden extraerse del trabajo de Marsden y Campbell (1984), se ha seguido usando una gran diversidad de indicadores, especialmente a medida que la metodología del análisis de redes sociales se ha popularizado en distintas disciplinas. A menudo, en la elección de estos indicadores no se hace referencia alguna a las distintas dimensiones posibles del concepto, lo que puede dar lugar a equívocos de distinto tipo. La tabla 2 tiene como objetivo mostrar esta gran diversidad de aproximaciones de forma resumida. El uso de unos u otros indicadores no es una cuestión menor, puesto que hacen variar la propia unidad de análisis, que va desde las características de la díada a la red como conjunto.

Tabla 2. Dimensiones e indicadores de la fuerza del vínculo en la literatura especializada

\begin{tabular}{l|l|l|l}
\hline $\begin{array}{c}\text { Dimensión y } \\
\text { sub-dimensiones }\end{array}$ & Referencia & $\begin{array}{c}\text { Dimensión de la fuerza } \\
\text { del vínculo }\end{array}$ & \multicolumn{1}{c}{ Indicador usado } \\
\hline Estructural & Friedkin (1980) & $\begin{array}{l}\text { Solapamiento de las } \\
\text { relaciones }\end{array}$ & $\begin{array}{l}\text { Proporción de relaciones } \\
\text { comunes entre cada par de } \\
\text { nodos. }\end{array}$ \\
$\begin{array}{l}\text { Expresiva (in- } \\
\text { tercambio de } \\
\text { apoyo) }\end{array}$ & Wellman (1982) & $\begin{array}{l}\text { Provisión de apoyo } \\
\text { emocional }\end{array}$ & Provisión de apoyo emocional \\
Social & $\begin{array}{l}\text { Lin, Vaighn y En- } \\
\text { sel (1981) }\end{array}$ & Homogeneidad social & $\begin{array}{l}\text { Homogeneidad en el estatus } \\
\text { social del contacto (homofilia } \\
\text { de estatus) }\end{array}$
\end{tabular}

EMPIRIA. Revista de Metodología de Ciencias Sociales. N. ${ }^{\circ}$ 26, julio-diciembre, 2013, pp. 149-174. ISSN: 1139-5737, DOI: 10.5944/empiria.26.7156 


\begin{tabular}{|c|c|c|c|}
\hline $\begin{array}{c}\text { Dimensión y } \\
\text { sub-dimensiones }\end{array}$ & Referencia & \begin{tabular}{|c|}
$\begin{array}{c}\text { Dimensión de la fuerza } \\
\text { del vínculo }\end{array}$ \\
\end{tabular} & Indicador usado \\
\hline $\begin{array}{l}\text { Estructural, so- } \\
\text { cial y expresiva } \\
\text { (tiempo inver- } \\
\text { tido) }\end{array}$ & $\begin{array}{l}\text { Mc Pherson, Po- } \\
\text { pielarz y Drobnic } \\
\text { (1992) }\end{array}$ & $\begin{array}{l}\text { Densidad, frecuencia } \\
\text { de contacto y distancia } \\
\text { socio-demográfica. }\end{array}$ & $\begin{array}{l}\text { Densidad de las conexiones } \\
\text { entre los alteri (relaciones } \\
\text { existentes /relaciones posi- } \\
\text { bles); distancia en años de } \\
\text { educación y titulación; fre- } \\
\text { cuencia de interacción. }\end{array}$ \\
\hline Social & $\begin{array}{l}\text { Stoloff, Glanville } \\
\text { y Bienenstock } \\
\text { (1999) }\end{array}$ & $\begin{array}{l}\text { Distancia social, tipo } \\
\text { de relación y acceso al } \\
\text { trabajo }\end{array}$ & $\begin{array}{l}\text { Lazos débiles: contactos no } \\
\text { familiares ni amigos que pro- } \\
\text { porcionan acceso al trabajo. } \\
\text { Puentes: contactos heterófilos } \\
\text { en "raza", género, barrio y } \\
\text { nivel de estudios. }\end{array}$ \\
\hline $\begin{array}{l}\text { Expresiva } \\
\text { (intensidad y } \\
\text { tiempo) }\end{array}$ & $\begin{array}{l}\text { Haines, Beggs y } \\
\text { Hulbert (1996, } \\
\text { 2008) }\end{array}$ & $\begin{array}{l}\text { Vínculos de apoyo con } \\
\text { personas similares }\end{array}$ & $\begin{array}{l}\text { "Densidad" operativizada } \\
\text { como intensidad expresiva y } \\
\text { duración de la relación. }\end{array}$ \\
\hline Expresiva & $\begin{array}{l}\text { Montgomery } \\
(1992)\end{array}$ & $\begin{array}{l}\text { Relaciones poco fre- } \\
\text { cuentes y "transitorias" } \\
\text { del mercado laboral }\end{array}$ & No o \\
\hline Estructural & $\begin{array}{l}\text { Baller, Robert y } \\
\text { Richardson, (2009) }\end{array}$ & $\begin{array}{l}\text { Difusión a través de } \\
\text { lazos no transitivos } \\
\text { (suicidio entre amigos } \\
\text { de amigos) }\end{array}$ & Índice de intransitividad \\
\hline $\begin{array}{l}\text { Expresiva } \\
\text { (tiempo e inten- } \\
\text { sidad) }\end{array}$ & Hans & $\begin{array}{l}\text { Frecuencia y cercanía } \\
\text { como intensidad emo- } \\
\text { cional }\end{array}$ & $\begin{array}{l}\text { Escalas tipo Likert de } 7 \text { pun- } \\
\text { tos. }\end{array}$ \\
\hline Estructural & $\begin{array}{l}\text { Shi, Adamic y } \\
\text { Strauss (2006). }\end{array}$ & $\begin{array}{l}\text { Transitividad de la } \\
\text { relación }\end{array}$ & $\begin{array}{l}\text { Lazos débiles: contactos que } \\
\text { no forman parte de ninguna } \\
\text { tríada cerrada. }\end{array}$ \\
\hline $\begin{array}{l}\text { Expresiva } \\
\text { (tiempo e inten- } \\
\text { sidad) }\end{array}$ & Atterton (2007) & $\begin{array}{l}\text { Cantidad de tiempo, y } \\
\text { cercanía como intensi- } \\
\text { dad emocional }\end{array}$ & $\begin{array}{l}\text { Duración de la relación, ha- } \\
\text { blar de asuntos personales y } \\
\text { frecuencia de contacto. }\end{array}$ \\
\hline $\begin{array}{l}\text { Expresiva } \\
\text { (tiempo) }\end{array}$ & $\begin{array}{l}\text { Gil de Zúñiga y } \\
\text { Valenzuela (2010) }\end{array}$ & $\begin{array}{l}\text { Tipo de relación que } \\
\text { une a ego y alter y fre- } \\
\text { cuencia de discusión }\end{array}$ & $\begin{array}{l}\text { Lazos débiles: escala de fre- } \\
\text { cuencia de discusión entre } \\
\text { conocidos y colegas. } \\
\text { Lazos fuertes: escala de fre- } \\
\text { cuencia de discusión entre } \\
\text { familiares y amigos. }\end{array}$ \\
\hline $\begin{array}{l}\text { Expresiva } \\
\text { (tiempo) }\end{array}$ & $\begin{array}{l}\text { Phithakkitnukoon, } \\
\text { Calabrese, Smore- } \\
\text { da y Ratti (2011) }\end{array}$ & Tiempo invertido & $\begin{array}{l}\text { Duración de las llamadas te- } \\
\text { lefónicas }\end{array}$ \\
\hline
\end{tabular}

Fuente: Elaboración propia. 
Como se comprueba en la tabla 2, a excepción de la propuesta de Gil de Zúñiga y Valenzuela (2010), la fuerza de los lazos se suele operativizar como un espectro con dos polos (los lazos fuertes y los lazos débiles), que se mide con un mismo indicador de intensidad. Este tipo de operativización se remonta al trabajo original de Granovetter (1973), para quien un lazo fuerte es aquel con quien se tiene una elevada frecuencia de contacto, y un lazo débil es un contacto al que vemos esporádicamente. En la misma línea, Wellman (1982) considera que un lazo fuerte es aquel con el que se intercambia apoyo emocional, y un lazo débil es una relación en la que no hay este tipo de apoyo; para Lin (2001) un lazo fuerte es aquel con quien tenemos una relación expresiva basada en la homogeneidad social, y un lazo débil es aquel con quién tenemos una relación instrumental basada en la heterogeneidad social.

Por otro lado, la tabla 2 pone de manifiesto el dominio de la dimensión expresiva sobre el resto de dimensiones a la hora de identificar los indicadores que desarrollan empíricamente la tesis de la fuerza de los lazos débiles. La presencia de las otras dimensiones puede obedecer, si seguimos los argumentos de Marsden y Campbell (1984), a la confusión entre indicadores del concepto teórico e indicadores que representan conceptos asociados o cercanos a la fuerza de los lazos, pero que estrictamente no pueden entenderse como medidas equivalentes. Este problema se produce, sin duda, como consecuencia de la falta de concreción en la definición original de Granovetter, pero también se origina probablemente por la falta de rigor y justificación de los indicadores elegidos en el proceso de operativización (véase Lazarsfeld, 1973).

En este sentido, algunos de los indicadores que Marsden y Campbell (1984) denominan "predictores", pueden entenderse también como variables explicativas de la fuerza del vínculo. Así, el tipo de relación (parentesco, amistad, vecindad, etcétera) puede concebirse -siguiendo a estos autores- como una característica que incide en la fuerza del vínculo, pero que no constituye en sí misma un atributo de la fortaleza del lazo. Del mismo modo, la distancia social o la homofilia puede entenderse como una característica que dificulta o facilita que un lazo sea fuerte o débil, pero difícilmente puede entenderse como un indicador directo de la fortaleza del lazo.

Estos razonamientos metodológicos que acabamos de realizar deberán contrastarse mediante análisis empíricos. Como se ha señalado en la introducción, los datos que utilizamos permiten realizar esta aproximación empírica en redes egocentradas de una extensión considerable. En las páginas siguientes se analizarán las relaciones entre variables de las tres dimensiones para comprobar su correspondencia y valorar en qué medida pueden resultar buenos indicadores, o no, del mismo constructo teórico.

\section{DATOS EMPLEADOS Y ESTRATEGIA DE ANÁLISIS}

Los datos con los que trabajamos proceden del proyecto I+D+I Estudio comparado de casos sobre la influencia mutua entre capital e integración sociales y la inserción, estabilidad, promoción y cualificación en el empleo (CASREDIN), subvencionado por el Ministerio de Educación y Ciencia. 
La encuesta de redes personales del proyecto ha emulado el muestreo de la Enquesta de Condicions de Vida i Hàbits de la Població (ECVHP) 2005, representativa a nivel de Cataluña, pero con un tamaño muestral mucho más reducido dados los costes asociados ${ }^{3}$ a la recogida de datos de redes personales. El muestreo fue estratificado y bietápico: la estratificación se ha hecho primero por localidades ${ }^{4}$ (Barcelona, Sant Feliu de Llobregat y Balaguer) y luego por colectivos de población, que responden al análisis factorial realizado sobre el censo de Cataluña del 2001 para el muestreo de la ECVHP (Lozares et al., 2011) y que distingue a la población por origen geográfico familiar y edad. ${ }^{5}$ La matriz utilizada se compone de 441 casos y contiene información relativa a 4 bloques de preguntas: un primer bloque de caracterización socio-demográfica de los ego, un segundo bloque en que se obtiene la red de 30 alteri (tamaño fijo), un tercer bloque de preguntas de caracterización socio-demográfica de cada alter mencionado, y por último, un bloque en que se obtienen las relaciones existentes entre cada par de alteri. Las redes personales se construyen a partir de relaciones de conocimiento mutuo, concretamente, la pregunta generadora solicita "30 personas (mayores de 18 años) que Usted conozca por su nombre (y viceversa) con las que ha tenido contacto al menos en los dos últimos años por cualquier medio de comunicación y que además pueda volver a contactar si fuese necesario".

Las variables con las que trabajamos se exponen a continuación:

- Proximidad afectiva en la relación. Ante cada contacto (alter) nombrado por ego se pidió que se valorara subjetivamente la proximidad de la relación en una escala del 1 al 5, cuyos extremos son 1: nada próximo y 5 : íntimo. Se han tomado los dos extremos, lo que supone una submuestra de 2726 contactos.

- Denominación de la relación. Variable nominal politómica construida a partir de la identificación del contexto de pertenencia de cada relación6.

- Polivalencia en la relación. Variable ordinal que distingue si una relación acumula múltiples contextos de relación, a partir de la suma de modalidades de la variable anterior.

- Intercambio de apoyo. Variables nominales dicotómicas que identifican a los contactos con quienes ego ha intercambiado apoyo, en el presente o

${ }^{3}$ Las personas encuestadas recibieron una pequeña gratificación por su participación en la encuesta, como modo de compensar el tiempo invertido en contestar el cuestionario.

${ }^{4}$ Se han realizado 150 encuestas en cada una de las ciudades.

5 Para mayor información sobre la construcción de la muestra, el anàlisis factorial en el origen del muestreo, la pregunta generadora y el resto del cuestionario, la elección de las unidades, etcétera, puede consultarse el artículo del proyecto CASREDIN en que se exponen todas estas cuestiones con todo lujo de detalles: Lozares et al. (2011).

${ }^{6}$ Las categorías que adopta la variable son: familia directa de ego (padre/madre, hermano/ hermana, hijo/hija), familia extensa, misma profesión de ego, misma empresa, misma asociación, mismo vecindario, mismo colegio de sus hijos (en caso de tenerlos). Las categorías de familia son excluyentes con el resto. 
en el pasado, en los siguientes ámbitos: trabajo, vivienda, salud y servicios médicos, escuela para los hijos u otros familiares, servicios sociales o administrativos, problemas personales, otros ámbitos.

- Ámbitos en el intercambio de apoyo. Variable nominal que identifica la polivalencia en el intercambio de apoyos a partir de la suma de las variables de apoyo anteriormente descritas. Contempla tres categorías (no hay intercambio de apoyo, apoyo en un ámbito, apoyo en dos ámbitos o más).

- Homofilia. Las variables sobre homofilia, nominales dicotómicas, se han construido a partir de la comparación de los valores de ego y alter en sendas variables atributivas y equivalentes, con las siguientes categorías:

- Categoría socio-profesional: alta, media, baja.

- Nivel de estudios finalizados: sin estudios, estudios obligatorios, estudios secundarios, estudios superiores.

- Duración de la relación. Variable continua que expresa la longevidad de la relación como proporción de la vida de ego, a partir de la división de los años de duración de la relación por la edad del entrevistado. Los valores próximos a 0 identifican las relaciones proporcionalmente más recientes, los valores próximos a 1 identifican a las relaciones de mayor duración.

- Transitividad local (intervalos). Variable ordinal a partir del indicador de la transitividad local. Comprende 4 categorías: Aislados (transitivi$\mathrm{dad}=0)$; conectados $[0.1,0.66]$; muy conectados $(0.66,1)$; en cliques (transitividad $=1$ ).

La estrategia de análisis se ha basado principalmente en el análisis de tablas de contingencia con $\mathrm{Chi}^{2}$ y V de Cramer para las comparaciones entre variables nominales, y en una comparación de medianas (Kruskal Wallis) para la variable continua (duración de la relación), que tiene una distribución muy alejada de la normal. También se han empleado análisis de frecuencias simples entre el conjunto de variables.

\section{RESULTADOS: COMPARACIÓN DE LOS INDICADORES DE LAS TRES DIMENSIONES}

En esta sección se realizan una serie de análisis con el objetivo de comparar las distintas dimensiones de la fuerza del vínculo que se han propuesto a nivel teórico (la expresiva, la estructural y la social), con los diferentes indicadores. Siguiendo la propuesta de Marsden y Campbell (1984), se toma como punto de partida a los contactos identificados como "nada próximos" para comparar el resto de indicadores. Si los resultados mostraran que los lazos expresivamente débiles también son socialmente más distantes, que 
están menos encastrados en la red de relaciones, y que intercambian menos apoyos, sería indicativo de que las tres medidas de la fuerza de los lazos tienden a converger, y por lo tanto, la conceptualización de sus dimensiones y la elección de indicadores sería equivalente en términos operativos. Pero, si por el contrario, los resultados mostraran divergencias en el comportamiento de los tres tipos de indicadores, se interpretaría como un apoyo a la argumentación realizada acerca de las diferencias sustantivas en las tres dimensiones del concepto, y la necesidad de justificar conceptualmente la elección de los indicadores para el análisis.

\subsection{Dimensión expresiva tipo de relación con ego}

En primer lugar, un análisis de frecuencias muestra que, fundamentalmente, las relaciones con las que no se siente ninguna proximidad a nivel afectivo (o lazos débiles en su dimensión expresiva) son personas que no pertenecen a la familia de ego $(84,4 \%)$ y algunas relaciones de familia extensa $(13,7 \%)$. El contexto laboral y el de vecinos es donde se encuentran más relaciones de este tipo.

Tabla 3. Distribución de los lazos afectivamente débiles según el tipo de relación con ego (porcentajes sobre el total de relaciones)

\begin{tabular}{cc}
\hline Tipo de relación & Porcentaje del total de relaciones \\
\hline Familia extensa & 13,7 \\
Misma profesión & 16,7 \\
Misma empresa & 11,8 \\
Misma asociación & 9,5 \\
Vecinos & 20,1 \\
Mismo colegio que los hijos & 2,3 \\
Otras relaciones sin definir & 25,9 \\
\hline
\end{tabular}

Fuente: Elaboración propia a partir de los datos de CASREDIN.

\section{Apoyo intercambiados con Ego}

A continuación se ha realizado una comparación por tablas de contingencia entre el intercambio de apoyos entre ego y sus contactos, para los distintos tipos de apoyo descritos anteriormente. Para realizar este análisis se ha seleccionado una sub-muestra del conjunto de datos, que incluye sólo a los contactos en los extremos de la variable de proximidad afectiva, los "nada próximos" y los "íntimos". El tamaño de la sub-muestra es de 2726 contactos. 
Tabla 4. Medidas de asociación en los análisis por tabla de contingencia entre la proximidad expresiva y el tipo de apoyos intercambiados

\begin{tabular}{ccc}
\hline Variables & $\begin{array}{c}\text { Valor de } \mathrm{X} 2 \text {, grados de libertad } \\
\mathrm{y} \text { valor } \mathrm{p} \text { asociado }\end{array}$ & $\begin{array}{c}\text { Valor de } \mathrm{V} \text { de Cramer y } \\
\text { valor } \mathrm{p} \text { asociado }\end{array}$ \\
\hline $\begin{array}{c}\text { Proximidad afectiva y apoyo } \\
\text { en cuestiones laborales }\end{array}$ & $190,09, \mathrm{gl}=1, \mathrm{p}=0,000$ & 0,$264 ; \mathrm{p}=0,000$ \\
$\begin{array}{c}\text { Proximidad afectiva y apoyo } \\
\text { en cuestiones de vivienda }\end{array}$ & $390,553, \mathrm{gl}=1, \mathrm{p}=0,000$ & 0,$379 ; \mathrm{p}=0,000$ \\
$\begin{array}{c}\text { Proximidad afectiva y apoyo } \\
\quad \text { en cuestiones de salud }\end{array}$ & $934,142, \mathrm{gl}=1, \mathrm{p}=0,000$ & 0,$585 ; \mathrm{p}=0,000$ \\
$\begin{array}{c}\text { Proximidad afectiva y apoyo } \\
\quad \text { en servicios sociales }\end{array}$ & $193,172, \mathrm{gl}=1, \mathrm{p}=0,000$ & 0,$267 ; \mathrm{p}=0,000$ \\
$\begin{array}{c}\text { Proximidad afectiva y apoyo } \\
\text { en problemas personales }\end{array}$ & $1198,467, \mathrm{gl}=1, \mathrm{p}=0,000$ & 0,$663 ; \mathrm{p}=0,000$ \\
\hline
\end{tabular}

Fuente: Elaboración propia a partir de los datos de CASREDIN.

La diferencia en el intercambio de apoyos con los contactos "nada próximos" y los "íntimos" es elevada y significativa, para todos los tipos de apoyo. Las V de Cramer denotan asociaciones fuertes y muy fuertes entre la proximidad afectiva y el intercambio de apoyo, es decir, entre la sub-dimensión de proximidad expresiva (intensidad afectiva) y la sub-dimensión de apoyo. La tabla 4 sintetiza los resultados de los test Chi2 y V de Cramer para cada análisis. Se aprecia una asociación muy estrecha entre la proximidad afectiva y el apoyo bajo las etiquetas de "problemas personales" y "cuestiones de salud", las cuales tienen un carácter más marcadamente expresivo, por lo que las dos sub-dimensiones tendrían una relación muy estrecha entre sí.

Tabla 5. Proporción de contactos "nada próximos" con quienes se intercambia uno o más tipos de apoyo

\begin{tabular}{cc}
\hline $\begin{array}{c}\text { Número de tipos de apoyo } \\
\text { intercambiados }\end{array}$ & $\begin{array}{c}\text { Porcentaje de contactos "nada } \\
\text { próximos" }\end{array}$ \\
\hline 0 & 57,5 \\
1 & 18,6 \\
2 & 12,0 \\
3 o más & 11,9 \\
\hline
\end{tabular}

Fuente: Elaboración propia a partir de los datos de CASREDIN.

Con respecto a la polivalencia en el intercambio de apoyos, en la figura 3 se observa que el $57 \%$ de los contactos "nada próximos" no comparte ningún tipo 
de apoyo con ego. Esos serían los contactos que se comportan del modo esperado para los lazos débiles. Sin embargo, hay un $43 \%$ que no lo hace. El 18,6\% comparte un tipo de apoyo con ego (los más habituales son trabajo y problemas personales) y el $23,9 \%$ restante comparten 2 o más tipos de apoyo. Es decir, dos de cada cinco alteri "nada próximos" intercambian apoyos de diverso tipo con ego, y casi una cuarta parte comparte varios tipos de apoyo.

Entre los apoyos con estos alteri "nada próximos" destacan el ámbito del trabajo, de los problemas personales, los de salud, y "otras cuestiones". Es decir, los apoyos intercambiados con estos contactos son bastante diversos.

Tabla 6. Porcentaje de contactos "nada próximos" con quienes se intercambia apoyo, según tipos de apoyo

\begin{tabular}{cc}
\hline Tipo de apoyo intercambiado & Porcentaje de contactos nada próximos \\
\hline Apoyo en cuestiones laborales & 21,3 \\
Apoyo en cuestiones de vivienda & 9,7 \\
Apoyo en cuestiones de salud & 10,4 \\
Apoyo en servicios sociales & 8,3 \\
Apoyo en problemas personales & 15,8 \\
Apoyo en otras cuestiones & 26,0 \\
\hline
\end{tabular}

Fuente: Elaboración propia a partir de los datos de CASREDIN.

\section{Polivalencia en la relación con ego}

El 55\% de los contactos "nada próximos" tiene una relación unidimensional con ego, es decir, o bien son vecinos, o bien contactos del entorno laboral, de la familia política, etcétera. El otro $45 \%$ de los contactos "nada próximos" tiene dos o más tipos de relación con ego, por ejemplo, son de la misma empresa y la misma profesión, o del mismo barrio y la misma asociación. El hecho de compartir más espacios o círculos (Feld, 1981) implica que una relación esté potencialmente más encastrada en la red, que se compartan más vínculos comunes. De este modo, se vulnera uno de los principios para que una relación sea un puente.

Tabla 7. Proporción de relaciones "nada próximas" según su polivalencia

\begin{tabular}{cc}
\hline $\begin{array}{c}\text { Polivalencia de las rela- } \\
\text { ciones }\end{array}$ & $\begin{array}{c}\text { Porcentaje de contactos "nada } \\
\text { próximos" }\end{array}$ \\
\hline I & 55,6 \\
2 & 38,9 \\
3 & 4,9 \\
4 & 0,6 \\
\hline
\end{tabular}

Fuente: Elaboración propia a partir de los datos de CASREDIN. 


\section{Duración de las relaciones}

Una prueba de Kruskal-Wallis muestra que hay una diferencia de medianas significativa en la duración de las relaciones con los contactos "íntimos" y con los "nada próximos" $\left(\mathrm{Chi}^{2}=522,76, \mathrm{gl}=1\right.$, valor $\left.\mathrm{p}=0,000\right)$. Como se aprecia en el diagrama de cajas (figura 7), las relaciones "íntimas" se concentran más entre las relaciones largas, y las "nada próximas" entre las relaciones recientes. Pero hay un $20 \%$ de relaciones dentro de los "nada próximos" que son de muy larga duración, es decir, uno de cada cuatro egos ha conocido a alteri afectivamente alejados durante la mitad de su vida o más.

Figura 3. Duración media de las relaciones con alteri "nada próximos" e "íntimos", expresado como proporción de la vida de ego

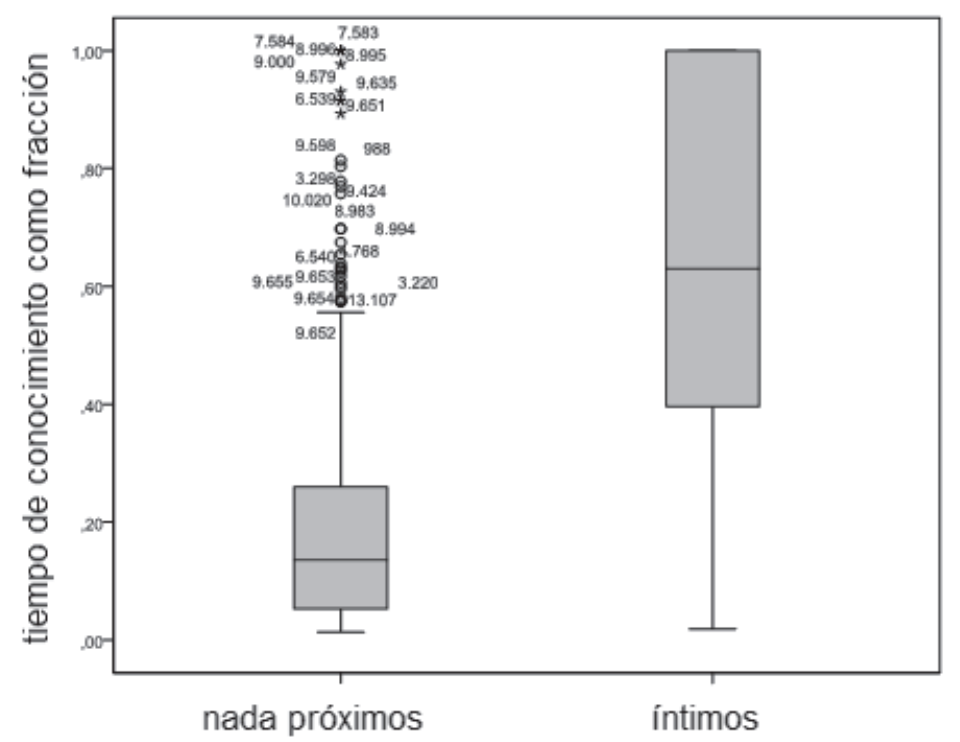

Fuente: Elaboración propia a partir de los datos de CASREDIN.

\subsection{Dimensión estructural transitividad local de los contactos}

La transitividad local es un indicador de posición de los contactos que informa sobre la medida en que un alter forma parte de tríadas cerradas (en las que todos los "vecinos"7 tienen relación directa entre sí), o si por el contrario, tiende a formar parte de subgrupos dispersos o relativamente aislados. La transitividad local es una variable continua, pero dada su distribución muy alejada

${ }^{7}$ Los otros alteri con los que tiene contacto directo.

EMPIRIA. Revista de Metodología de Ciencias Sociales. N. ${ }^{\circ}$ 26, julio-diciembre, 2013, pp. 149-174. ISSN: 1139-5737, DOI: 10.5944/empiria.26.7156 
de la normalidad, la hemos convertido en una variable categórica para facilitar su tratamiento.

Al comparar la transitividad local entre contactos "íntimos" y "nada próximos" (mediante una tabla de contingencia), se aprecia una pauta de polarización, opuesta para ambos grupos: los nada próximos están sobre-representados entre los aislados y los que forman parte de cliques. Es decir, son lazos que, o bien se mantienen activos a causa de su estrecha relación con el resto de la red, o bien se tienden a aislar. Por el contrario, los contactos "íntimos" tienden a estar conectados con el resto de los contactos de la red, sin estar necesariamente en relación con todos los otros lazos. La figura 6 sintetiza esta pauta polarizada.

\section{Figura 4. Distribución de la transitividad local según intensidad expresiva de la relación (frecuencias relativas)}

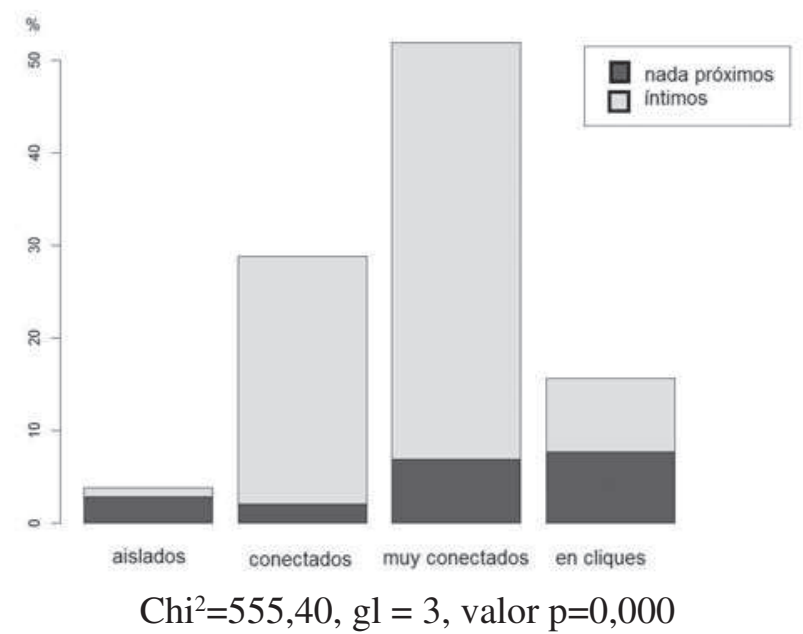

Fuente: Elaboración propia a partir de los datos de CASREDIN.

La distribución de la transitividad local respecto a la intensidad del contacto estaría mostrando dos tipos de lazos débiles: un primer tipo más aislado de la red de contactos, de modo coherente con lo previsto por Granovetter (1973), y un segundo tipo de contactos bien encastrados en la red de relaciones, mostrando una vez más las inconsistencias internas de la categoría "nada próximos".

\subsection{Dimensión social lazos débiles como interacciones heterófilas}

A continuación, comparamos la proporción de contactos homófilos que son "íntimos" o "nada próximos".

EMPIRIA. Revista de Metodología de Ciencias Sociales. N. ${ }^{\circ}$ 26, julio-diciembre, 2013, pp. 149-174. ISSN: 1139-5737, DOI: 10.5944/empiria.26.7156 
A diferencia de lo que cabría esperar bajo los supuestos de Lin (2001), así como otros autores que vinculan la homofilia a los lazos fuertes (véase Haines, Beggs y Hulbert, 1996; 2008) no se observa una pauta clara que diferencie a los contactos expresivamente débiles y a los fuertes en lo que a homofilia se refiere. Las $\mathrm{V}$ de Cramer denotan relaciones insignificantes entre las variables (inferiores a 0,07$)$. Por lo tanto, concluiremos que la intensidad expresiva de la relación no está realmente relacionada con la similitud socio-demográfica del contacto. Los amigos "íntimos" son igual de (poco) parecidos que los contactos afectivamente distantes.

Figura 5. Comparación de lazos homófilos en categoría profesional y nivel de estudios entre "íntimos" y "nada próximos" (proporciones).
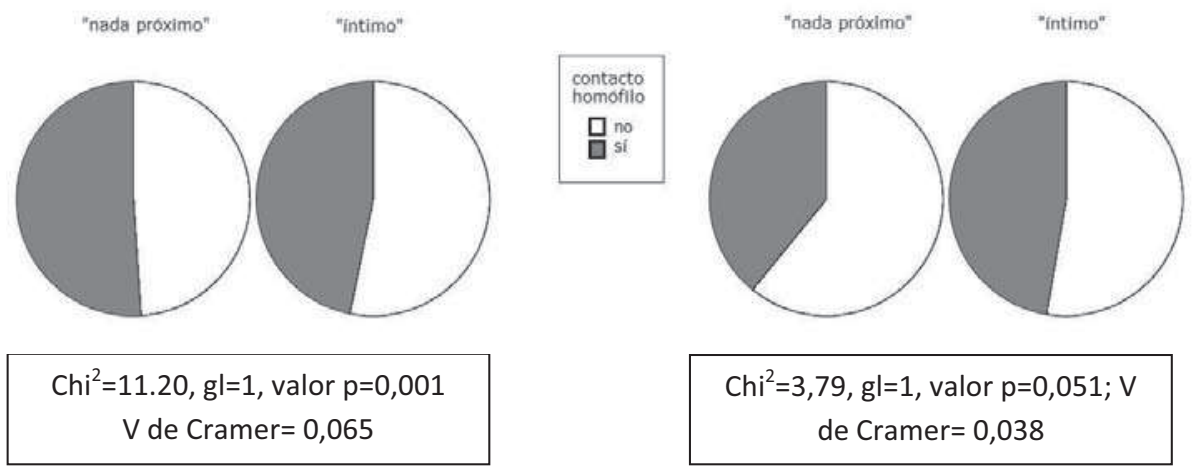

Fuente: Elaboración propia a partir de los datos de CASREDIN.

Este dato tiene una lectura relevante en términos de la comprensión de la homofilia: por una parte, si los contactos "íntimos" son igual de homófilos que los nada próximos, cabría pensar que la homofilia es algo que afecta a las relaciones de los individuos independientemente de sus preferencias, y por lo tanto, de sus elecciones. Bajo esta comprensión, los filtros estructurales sobre los círculos de relación (Feld, 1981) tienen un efecto manifiesto, incluso sobre las relaciones con las que no tenemos proximidad. Este sería un argumento a favor de la perspectiva estructural dentro del debate sobre el origen de la homofilia en las relaciones (Kossinets y Watts, 2009).

Sin embargo, es extensa la literatura que constata los efectos de selección de las relaciones con aquellos más similares (Hamm, 2000; Portes y Sensenbrenner 1993; Mollica, Gary y Trevino, 2003, citados por Lozares y Verd, 2011; Kossinets y Watts, 2009). Esto nos lleva a pensar que la operativización de la homofilia sobre una característica tan genérica como la categoría socio-profesional, al estilo de Lin (2001), no permite realmente hablar de una dimensión afectiva de las relaciones. Es decir, que no sería erróneo suponer que se prefieran a personas similares para las relaciones más próximas, sino pensar que esta selección se da en base a una categoría tan genérica como el estatus socio-laboral. A nuestro 
entender, la homofilia por categoría socio-profesional responde más a los efectos contextuales que a las motivaciones de los agentes, como sugiere Lin (2001).

Los resultados obtenidos utilizando este indicador nos llevan a afirmar que si bien la homofilia puede ser un buen indicador de la distancia social, haría falta argumentar con mayor detalle su uso como medida de la fuerza del vínculo. Posiblemente características atributivas más vinculadas a la dimensión expresiva de la fuerza de los vínculos, como por ejemplo unas aficiones similares, unos valores o actitudes parecidos, o incluso una ideología cercana, podrían ser útiles como aproximación a los lazos fuertes.

\section{CONCLUSIONES}

\section{Síntesis de los resultados}

El análisis de datos realizado nos ha permitido llegar a todo un conjunto de resultados que resumimos a continuación. En primer lugar, en relación con las múltiples dimensiones que componen el concepto de Granovetter (1973) de "fuerza de los lazos", se ha comprobado que el indicador de proximidad afectiva correlaciona con el resto de potenciales indicadores de una relación fuerte del modo previsto por la teoría: las relaciones íntimas se ayudan más, en más ámbitos, tienen más tipos de relación con ego, están bien conectados con su entorno, y son relaciones de más larga duración.

Sin embargo, hay una proporción grande de lazos con una valoración de proximidad afectiva baja que se comporta de un modo inconsistente con lo previsto por la teoría, por lo que cabría suponer que esta medida incluye distintos formatos de relación, como por ejemplo relaciones superficiales, relaciones puntuales, recientes, conflictivas, etcétera. Las inconsistencias observadas se detallan a continuación: 1) Los lazos débiles en nuestra muestra son principalmente contactos laborales y vecinos. También familia extensa. Es decir, son relaciones con cierta cotidianidad, y potencialmente encastradas en un grupo de conocidos más extenso, que difícilmente constituirán componentes aislados. 2) El 45\% tiene más de un tipo de relación con ego, es decir, casi la mitad son relaciones polivalentes en las que se superponen distintos focos de relación (Feld, 1981), lo cual lleva a suponer que se comparten más relaciones y que no serán puentes. 3) El $45 \%$ intercambia alguna forma de apoyo con ego, incluyendo formas de apoyo instrumental, como por ejemplo las relacionadas con el trabajo. Sin embargo, hay un $25 \%$ de estos contactos que intercambia dos o más tipos de apoyo, denotando de nuevo una situación de polivalencia. Cabría entonces plantear la hipótesis de que una valoración baja en la proximidad afectiva pueda confundir los lazos débiles o las relaciones superficiales, con las relaciones conflictivas, o relaciones expresivamente fuertes pero deterioradas. Esto plantearía la necesidad de revisar la adecuación del indicador. 4) Un 20\% de estos contactos nada próximos son relaciones de larga duración, a pesar de que los lazos débiles tienden a perderse si no se apoyan en una red común de relaciones, u otro contexto insti- 
tucional de encastramiento, especialmente entre las clases trabajadoras (Allan, 1977). 5) Una elevada proporción de las relaciones "nada próximas" son muy transitivas, por lo que no pueden ser puentes.

Por otro lado, no observamos en nuestros datos diferencias significativas en la homofilia entre los lazos con una proximidad afectiva baja y los lazos "íntimos". Esta es una información relevante en la medida que parece entrar en contradicción con la literatura que ha detectado la preferencia psicológica por la interacción con aquellos similares a uno mismo. Sin embargo, las características sobre las que versa la homofilia con las que hemos trabajado (y las propuestas por Lin, 1981) son características socio-demográficas a un nivel muy genérico, distintas a las empleadas en la literatura que trabaja con las preferencias por los similares. En cambio, en las características que versan sobre la posición de los individuos dentro de la estructura social, cabría pensar que los filtros estructurales sobre los círculos de relación (Feld, 1981) ejercen su efecto incluso sobre las relaciones con las que no tenemos proximidad. Es decir, independientemente de las preferencias individuales, las personas con las que tenemos relación, ya sea ésta más o menos íntima, proceden de posiciones sociales similares.

\section{Algunos elementos de reflexión}

Las inconsistencias halladas entre teoría y resultados empíricos nos llevan a pensar que existe un problema en la definición del concepto "fuerza del vínculo". La gran diversidad de indicadores empleados en la literatura, que en el artículo hemos clasificado en tres dimensiones, expresiva, social y estructural, ya deja intuir este problema de definición del concepto. Los análisis realizados confirman este problema de definición: algunas de las correlaciones que la teoría predice no se dan en los datos. En nuestro análisis, los datos muestran que las dimensiones expresiva y social son independientes entre sí, a diferencia de lo que se supone en planteamientos precedentes como el de Nan Lin (1999a).

El análisis de los datos muestra también que las distintas subdimensiones expresivas tienen asociaciones fuertes entre sí, tal como predice la teoría. Los resultados obtenidos indican que la variable de proximidad afectiva de la relación recoge correctamente los lazos fuertes. Estas relaciones quedan bien definidas y correlacionan coherentemente con el resto de indicadores de la dimensión expresiva. Sin embargo, los lazos débiles están peor caracterizados por esta variable, se mezclan y confunden con relaciones conflictivas, relaciones recientes, relaciones de indiferencia, etcétera. Es decir, la operativización de los lazos débiles como ausencia de intensidad afectiva acarrea ambigüedades en la identificación de las relaciones.

Dados estos resultados, nos inclinamos a pensar que las dimensiones expresiva y social serían mejor abordadas como nociones independientes la una de la otra, y no como dimensiones del concepto de "fortaleza del vínculo". En este sentido, los datos parecen confirmar que las variables vinculadas a la dimensión social no son en realidad un buen indicador del concepto propuesto por Granovetter. El tipo de análisis realizado no permite detectar si existe una relación de dependencia (en la línea que hemos defendido anteriormente a nivel teórico) 
entre las variables asociadas a la dimensión social y las asociadas a la dimensión expresiva. Sin embargo, sí parece posible afirmar, a partir de los resultados y de la revisión teórica realizada, que la dimensión social estaría fundamentalmente vinculada a la noción de puente (por lo tanto constituiría una característica formal fruto de la estructura de la red de contactos) y que la dimensión expresiva estaría más vinculada a la noción de fortaleza del lazo, siendo por lo tanto un atributo relativo al contenido (más profundo o más superficial, más frecuente o más esporádico) de la relación, y no a sus características formales.

Finalmente, resulta interesante comentar un resultado no estrictamente vinculado a los objetivos iniciales del análisis, pero que sí está vinculado al abordaje empírico de la fortaleza de los lazos. Como se ha comentado, la encuesta de redes personales utilizada como fuente de los datos solicitaba información sobre treinta alteri a cada uno de los egos entrevistados. Este procedimiento, propuesto por McCarty (2002) permite, efectivamente, recoger una mayor diversidad de tipos de relaciones y de vínculos de distinta intensidad. Sin embargo, el análisis realizado muestra que no se puede asumir que estos lazos débiles sean puentes. Los lazos débiles así recogidos, a diferencia de otras preguntas generadoras más centradas en las relaciones fuertes, se mezclan con el conjunto antes mencionado de relaciones conflictivas, relaciones recientes o deterioradas, cuestión que vuelve a remitirnos a la importancia de tratar como nociones independientes la dimensión social (y estructural) de la fuerza de los lazos y la dimensión expresiva.

\section{Límites y líneas futuras de investigación}

La limitación más evidente del trabajo realizado obedece al hecho de haber abordado sólo superficialmente la dimensión que hemos denominado estructural. $\mathrm{Al}$ trabajar con redes egocentradas resulta imposible identificar correctamente la estructura completa de la red de relaciones, y en concreto los agujeros estructurales. Repetir estas comparaciones de indicadores con datos provenientes de redes completas ayudaría a mejorar la aproximación aquí propuesta.

Por otro lado, un análisis más profundo de la asociación o no entre la dimensión expresiva y la social requeriría utilizar indicadores de homofilia más centrados en variables de tipo más expresivo, como las aficiones o los valores o actitudes. En nuestra investigación estos indicadores no se pudieron construir al no haberse recogido dichas variables en la encuesta utilizada. Una futura línea de investigación se podría orientar en esta dirección.

\section{BIBLIOGRAFÍA}

ALLAN, G. (1977). "Class Variation in friendship patterns". The British Journal of Sociology. Vol. 28, n. ${ }^{\circ} 3$, pp. 389-393.

ATRIA, R. (2003). "Capital social: concepto, dimensiones y estrategias para su desarrollo", en ATRIA R.; SILES M.; ARRIAGADA I.; ROBISON L.J.; WHITEFORD S.: Capital social y reducción de la pobreza en América Latina y el Caribe: en busca de un nuevo paradigma, Libros de la CEPAL, Santiago de Chile. 
ATTERTON, J. (2007). “The 'Strength of Weak Ties': Social Networking by Business Owners in the Highlands and Islands of Scotland". Sociologia Ruralis, vol. 47, n. 3. [Edición digital].

BALLER, R.; RICHARDSON, K. (2009). "The « Dark Side » of the Strength of Weak Ties: The Diffusion of Suicidal Thoughts", Journal of Health and Social Behavior, Vol. 50, pp. 261-276.

BOTT, E. (1957). Family and social network: roles, norms, and external relationships in ordinary urban families. London : Tavistock, [1968].

BURT, R.S. (1982). Toward a Structural Theory of Action: Network Models of Social Structure, Perception and Action. Nueva York: Academic Press.

BURT, R.S. (1992). Structural holes: the social structure of competition. London: Cambridge Eds.

COHEN, J. (1977). "Sources of Peer Group Homogeneity". Sociology of Education, Vol. 50, No. 4 (Oct., 1977), pp. 227-241.

DE FEDERICO DE LA RUAA, A. (2011) "La amistad como apoyo desde una perspectiva comparada" en Felix Requena (ed.) Las redes de apoyo social. Aranzadi: Editorial Civitas.

FELD, S.L. (1981). "The focused organization of social ties". American Journal of Sociology. Vol.86, n. ${ }^{\circ}$ 5. pp. 1015-1035.

FORSÉ, M. (1997). “Capital social et emploi”. L Lné e sociologique, vol. 47, n. o 1, pp. 143-181.

GIL DE ZÚNIIGA, H.; VALENZUELA, S. (2010). "The mediating path to a stronger citizenship: online and offline networks, weak ties, and civic engagement". Соттиnication Research, vol. 38, pp. 397- 421.

GRANOVETTER, M. (1973). "The strength of weak ties". The American Journal of Sociology. Vol. 78, n. ${ }^{\circ} 6$ (Mayo), pp. 1360-1380.

GRANOVETTER, M. (1974). Getting a job: a study of contacts and careers. Cambridge, Mass: Harvard University Press.

GRANOVETTER, M. (1983). "The strength of weak ties: a network theory revisited". Sociological Theory, Vol. 1, pp. 201-233.

GRANOVETTER, M. (1992). "Economic Action and Social Structure: The Problem of Embeddedness", en Mark Granovetter y Richard Swedberg (ed.): The Sociology of Economic Life. Boulder, Colorado: Westview Press.

FRIEDKIN, N. (1980). “A test of structural features of Granovetter's Strength of Weak Ties Thory". Social Networks, Vol.2, pp. 411-422.

HAINES, V.; HURLBERT, J.; BEGGS, J. (1996). "Exploring the Determinants of Support Provision: Provider Characteristics, Personal Networks, Community Contexts, and Support Following Life Events. Journal of Health and Social Behavior, Vol. 37 , No. 3 (Sep. 1996), pp. 252-264.

HAINES, V.; HURLBERT, J.; BEGGS, J. (2008).“'Contextualizing Health outcomes: do effects of network structure differ for women and men?". Sex Roles, n. ${ }^{\circ}$ 59, pp. 164-175.

HANNEMAN, R.; RIDDLE, M. (2005). Introduction to social network methods. Riverside, California: University of California Riverside.

HANSEN, M. (1999). "The Search-Transfer Problem: The Role of Weak Ties in Sharing Knowledge across Organization Subunits". Administrative Science Quarterly, vol. $44,{ }^{\circ}{ }^{\circ}$, pp. 82-111.

KANDEL, DB. 1978. Homophily, selection and socialization in adolescent friendships. American Journal of Sociology. Vol. 84, pp. 427-36 
KOSSINETS, G.; WATTS, D. (2009). "Origins of Homophily in an Evolving Social Network". American Journal of Sociology, Vol. 115, n. . 2, pp. 405-450.

LAZARSFELD, P.F. (1973) "De los conceptos a los índices empíricos". En Boudon, R. y Lazarsfeld, P.F. (comps.): Metodología de las Ciencias Sociales. Conceptos e índices. Barcelona: Laia, pp. 35-46.

LIN, N. (1999a). "Social Networks and status attainment". Annual Review of Sociology, vol. 25 , pp. $467-487$.

LIN, N. (1999b). "Building a network theory of social capital". Connections. Vol. 22, $\mathrm{n}^{\circ} 1: 28-51$

LIN, N. (2001). Social Capital: a theory of structure and action. New York: Cambridge University Press.

LIN, N.; ENSEL, W.; VAUGHN, J. (1981). "Social resources and strength of ties: structural factors in occupational status attainment". American Sociological Review, Vol. 46, n. ${ }^{\circ}$ 4, pp. 393-405.

LOZARES, C.; LÓPEZ-ROLDÁN, P.; VERD, J.M.; MARTÍ, J.; BOLÍBAR, M.; CRUZ, I.; MOLINA, J.L. (2011). "El análisis de la Cohesión, Vinculación e Integración sociales en las encuestas EgoNet", REDES-Revista hispana para el análisis de redes sociales, vol. 20 \#4 (http://revista-redes.rediris.es) [edición digital].

LOZARES, C.; VERD, J.M. (2011). "De la homofilia a la cohesión social y viceversa". REDES-Revista hispana para el análisis de redes sociales. Vol. 20, \#2. [edición digital].

LOZARES, C.; VERD, J.M.; LÓPEZ-ROLDÁN, P.; MARTÍ, J.; MOLINA, J.L. (2011). "Cohesión, Vinculación e Integración sociales como formas de Capital social", $R E$ DES-Revista hispana para el análisis de redes sociales, vol. 20 \#1 (http://revistaredes.rediris.es) [edición digital].

MARSDEN, P.; CAMPBELL, K. (1984). "Measuring tie strength". Social forces, vol. $63,{ }^{\circ}{ }^{\circ}$, pp. $482-501$.

MCCARTY, C. (2002). "Structure in Personal Networks". Journal of social structure, vol. 3, n. $^{\circ} 1$. [edición digital]

MCPHERSON, M.J. ; POPIELARZ, P. ; DROBNIC, S. (1992). "Social Networks and Organizational Dynamics”. American Sociological Review, Vol. 57, n. ${ }^{\circ}$ 2, pp. 153-170.

MCPHERSON, M.J.; SMITH-LOVIN, L.; COOK, J.M. (2001). "Birds of a feather: Homophily in social networks". Annual Review of Sociology, vol. 27, pp. 415-44.

MONTGOMERY, J. (1992). "Job search and network composition: implications of the strength of weak ties hypothesis". American Sociological Review, vol. 57, n. ${ }^{\circ}$, pp. 586-596.

PHITHAKKITNUKOON, S.; CALABRESE, F.; SMOREDA, Z.; RATTI, C. (2011). Out of Sight Out of Mind $\bigotimes$ How our mobile social network changes during migration. Comunicación presentada en el Third IEEE International Conference on Social Computing, disponible en http://web.mit.edu/santi/www/socialcom11_migration.pdf.

RAPOPORT, A. (1957). "Contributions to the Theory of Random and Biased Nets." Bulletin of Mathematical Biophysics, vol. 19, pp. 257-277.

SHI, S.; ADAMIC, L.; STRAUSS, M. (2006). "Networks of strong ties". Archivos de la Cornell University Library [arXiv:cond-mat/0605279 [cond-mat.stat-mech]], disponible en http://arxiv.org/abs/cond-mat/0605279.

STOLOFF, J.; GLANVILLE, J.; BIENENSTOCK, E. (1999). "Women's participation in the labor force: the role of social networks". Social Networks, vol. 21, pp. 91-108.

WELLMAN, B. (1982). "Studying personal communities". En P. Marsden \& N. Lin (Ed.), Social structure and network analysis (pp. 61-80). Beverly Hills, CA: Sage. 
WELLMAN, B. (1990). "Different Strokes from Different Forlks: Community Ties and Social Support". American Journal of Sociology, Vol. 96, n. ${ }^{\circ} 3$, pp. 558-588.

WOOLCOCK, M.; NARAYAN N. (2000). "Social Capital: Implications for Development Theory, Research and Policy", The World Bank Research Observer, vol. 15, n. ${ }^{\circ}$, pp. $225-249$. 